\title{
The genome of Naegleria lovaniensis, the basis for a comparative approach to unravel pathogenicity factors of the human pathogenic amoeba N. fowleri
}

Nicole Liechti ${ }^{1,2,3}$, Nadia Schürch ${ }^{2}$, Rémy Bruggmann ${ }^{1}$ and Matthias Wittwer ${ }^{2^{*}}$

\begin{abstract}
Background: Members of the genus Naegleria are free-living eukaryotes with the capability to transform from the amoeboid form into resting cysts or moving flagellates in response to environmental conditions. More than 40 species have been characterized, but only Naegleria fowleri ( $N$. fowleri) is known as a human pathogen causing primary amoebic meningoencephalitis (PAM), a fast progressing and mostly fatal disease of the central nervous system. Several studies report an involvement of phospholipases and other molecular factors, but the mechanisms involved in pathogenesis are still poorly understood. To gain a better understanding of the relationships within the genus of Naegleria and to investigate pathogenicity factors of $\mathrm{N}$. fowleri, we characterized the genome of its closest non-pathogenic relative N. lovaniensis.
\end{abstract}

Results: To gain insights into the taxonomy of Naegleria, we sequenced the genome of $\mathrm{N}$. lovaniensis using long read sequencing technology. The assembly of the data resulted in a $30 \mathrm{Mb}$ genome including the circular mitochondrial sequence. Unravelling the phylogenetic relationship using OrthoMCL protein clustering and maximum likelihood methods confirms the close relationship of $\mathrm{N}$. lovaniensis and $\mathrm{N}$. fowleri. To achieve an overview of the diversity of Naegleria proteins and to assess characteristics of the human pathogen N. fowleri, OrthoMCL protein clustering including data of N. fowleri, N. lovaniensis and N. gruberi was performed. GO enrichment analysis shows an association of N. fowleri specific proteins to the GO terms "Membrane" and "Protein Secretion."

Conclusion: In this study, we characterize the hitherto unknown genome of N. lovaniensis. With the description of the $30 \mathrm{Mb}$ genome, a further piece is added to reveal the complex taxonomic relationship of Naegleria. Further, the whole genome sequencing data confirms the hypothesis of the close relationship between N. fowleri and N. lovaniensis. Therefore, the genome of $\mathrm{N}$. lovaniensis provides the basis for further comparative approaches on the molecular and genomic level to unravel pathogenicity factors of its closest human pathogenic relative $N$. fowleri and possible treatment options for the rare but mostly fatal primary meningoencephalitis.

Keywords: Naegleria lovaniensis, Naegleria fowleri, PacBio sequencing, Genome de novo assembly, Primary amoebic meningoencephalitis, Comparative genomics

\footnotetext{
* Correspondence: matthias.wittwer@babs.admin.ch

${ }^{2}$ Biology Division, Spiez Laboratory, Federal Office for Civil Protection,

Austrasse, Spiez, Switzerland

Full list of author information is available at the end of the article
}

(c) The Author(s). 2018 Open Access This article is distributed under the terms of the Creative Commons Attribution 4.0 International License (http://creativecommons.org/licenses/by/4.0/), which permits unrestricted use, distribution, and reproduction in any medium, provided you give appropriate credit to the original author(s) and the source, provide a link to the Creative Commons license, and indicate if changes were made. The Creative Commons Public Domain Dedication waiver (http://creativecommons.org/publicdomain/zero/1.0/) applies to the data made available in this article, unless otherwise stated. 


\section{Background}

Naegleria spp. are free-living amoebas of the class Heterolobosea and are found in soil and fresh water sources all over the world. As amoeboflagellates, they are able to transform under changing environmental conditions from the amoeboid form into fast moving flagellates or resting cysts [1]. Based on the analysis of internal transcribed spacer sequences located on the ribosomal DNA, over 40 species have been characterized [2]. So far only one species, $N$. fowleri is known as a human pathogen causing primary amoebic meningoencephalitis (PAM), a fast progressing and mostly fatal disease of the central nervous system [3]. Infections with $N$. fowleri occur when contaminated water, for example during swimming or ritual nose cleansing, enters the nose and the amoeba find its way along the olfactory nerves to the brain by crossing the cribriform plate $[4,5]$. The progression of the disease is rapid, and most patients die within 14 days. In many cases, the diagnosis is made post mortem [6]. While the worldwide prevalence is low, in several countries (e.g. Pakistan) the number of reported cases increased in the last few years [7]. Several studies report an involvement of phospholipases, proteases and other molecular factors, but the mechanisms involved in the pathogenesis are poorly understood. Although the genome of $N$. fowleri was published in 2014 in connection with a comparative proteomics study of high versus low pathogenic amoebae [8], there is still little known about Naegleria on the genomic and transcriptomic level. One of the best characterized species is the non-pathogenic and non-thermotolerant $N$. gruberi. It serves as a model for basal body and flagellar assembly processes and its genome was published in 2008 [9]. Since $N$. gruberi is well studied as a model organism, several groups assessed pathogenicity factors using a comparative approach, for example by comparing protease activities [10] or membrane glycoconjugates [11] between the human pathogenic $N$. fowleri and the non-pathogenic $N$. gruberi. The suitability of $N$. gruberi as a close non-pathogenic model is questioned by the work of Herman et al. [12] analysing the mitochondrial sequences and a $60 \mathrm{~kb}$ nuclear segment of $N$. fowleri and $N$. gruberi. The comparison of these genomic features showed less synteny than expected [12]. Therefore, accessing virulence factors by an interspecies comparison of $N$. fowleri and $N$. gruberi on a molecular and genomic level may result in misleading findings. Moreover, phylogenetic analysis of Naegleria based on ribosomal DNA and internal transcribed spacer sequences gives evidence, that $N$. lovaniensis is closer related to $N$. fowleri. Due to the high sequence similarity, it is even probable that $N$. fowleri and $N$. lovaniensis evolved from a common ancestor [2]. Summarized, there is still a lack of knowledge regarding phylogenetic relationships as well as the diversity on a genomic level. However, to unravel mechanisms involved in pathogenesis, knowledge of the genomic structure and taxonomy of the Genus Naegleria are crucial. In the last few years, Next Generation Sequencing (NGS) Technologies evolved rapidly. Besides short read sequencing, new methods with substantially increased read length such as Single Molecule Real Time sequencing have gained more importance. With the ability of spanning repetitive sequences, long reads provide new possibilities in the field of de novo genome assembly. New bioinformatics tools for genome de novo assembly have also been established [13, 14]. Long read assemblers implement approaches that consider the heterozygosity of genomes and comprise algorithms for the correction of sequencing errors. They are powerful tools for de novo assembly of eukaryotic genomes. To gain insights into the diversity of Naegleria focusing on the pathogenicity of $N$. fowleri, we characterize the genome of the close non-pathogenic relative $N$. lovaniensis. With the assembly of the $N$. lovaniensis genome, a further reference is added to the class of Heterolobosea and provides insights into the complex phylogenetic structure of the genus of Naegleria. The sequencing data support the close relationship of $N$. fowleri and $N$. lovaniensis. Furthermore, the characterization of $N$. lovaniensis provides the basis for further comparative approaches on the molecular and genomic level to unravel pathogenicity factors of $N$. fowleri. Analysis of $N$. fowleri specific protein clusters identified members of Rab and Rho small family GTPases, which are involved in vesicular trafficking and cytoskeletal reorganization. Furthermore, our data highlight the importance of actin adhesion structures and secretory processes during $N$. fowleri pathogenesis.

\section{Methods}

Cultivation of $N$. lovaniensis

$N$. lovaniensis trophozoites (ATCC \#30569) were cultivated at $36{ }^{\circ} \mathrm{C}$ in different media types according to Burri et al. [15] using Nunclon $^{\mathrm{Tm}} \Delta$ Surface cell culture flasks (Thermo Fisher Scientific, Allschwil, Switzerland). Nelson's Medium contains $0.1 \%(w / v)$ Liver Hydrolysate (Sigma, Buchs, Switzerland), 0.1\% (w/v) D-(+)-glucose (Sigma), and $10 \%(v / v)$ fetal calf serum in Page's amoeba saline $\left(2 \mathrm{mM} \mathrm{NaCl}, 16 \mu \mathrm{M} \mathrm{MgSO}_{4}, 27.2 \mu \mathrm{M} \mathrm{CaCl}_{2}, 1 \mathrm{mM}\right.$ $\left.\mathrm{Na}_{2} \mathrm{HPO}_{4}, 1 \mathrm{mM} \mathrm{KH} \mathrm{PO}_{4}\right)$. PYNFH medium consists of $1 \%(w / v)$ Bacto peptone (BD Biosciences), 1\% (w/v) yeast extract (BD Biosciences), 0.1\% (w/v) yeast ribonucleic acid (Sigma), $15 \mathrm{mg}$ folic acid (Sigma) and $1 \mathrm{mg}$ haemin (Sigma) supplemented with $10 \%(v / v)$ fetal calf serum in $133 \mathrm{mM} \mathrm{KH} \mathrm{PO}_{4}, 176.1 \mathrm{mM} \mathrm{Na} \mathrm{HPO}_{4}$. As a third media type, PYNFH medium was supplemented with $0.1 \%(\mathrm{w} / \mathrm{v})$ Liver Hydrolysate (Sigma, Buchs, Switzerland).

\section{Sequencing of genomic DNA}

For DNA isolation, $N$. lovaniensis was cultivated in Nelson's Medium, trophozoites were detached from culture 
flask using a cell scraper and harvested by centrifugation followed by three washing steps using Dulbecco's phosphate-buffered saline (PBS, Sigma). DNA was extracted using DNeasy Blood and Tissue Kit (Qiagen, Basel, Switzerland) according to the manufacturer's protocol. Any remaining RNA was digested using RNase A (Qiagen). The DNA was eluted in $100 \mu \mathrm{l}$ EB Buffer (10 mM Tris-Cl, $\mathrm{pH} 8.5$, Qiagen) preheated to $70{ }^{\circ} \mathrm{C}$. Total DNA was quantified using the Qubit 2.0 Fluorometer with the Qubit dsDNA HS Assay Kit (Thermo Fisher Scientific). Further, the quality was analysed using the Agilent 2100 Bioanalyzer system with the Agilent DNA 12000 Kit (Agilent Technologies, Basel, Switzerland). High molecular-weight DNA $(18 \mu \mathrm{g})$ was sent to the Functional Genomic Centre (Zurich, Switzerland) for library preparation and PacBio Sequencing of 10 SMRT cells using P6C4 chemistry on RSII platform.

\section{Sequencing of RNA}

For total RNA isolation, $N$. lovaniensis was cultivated in the media Nelson, PYNFH and PYNFH supplemented with Liver Hydrolysate. Trophozoites were harvested in the late log phase by centrifugation and washed three times using PBS. For cell disruption, trophozoites were resuspended in $750 \mu \mathrm{l}$ QIAzol lysis reagent (Qiagen) and homogenized in the TissueLyser (Qiagen) for $3 \mathrm{~min}$ at $25 \mathrm{~Hz}$. After incubation at room temperature for $5 \mathrm{~min}$, $150 \mu \mathrm{l}$ chloroform (Sigma) was added to the sample followed by centrifugation for $15 \mathrm{~min}$ at $12,000 \mathrm{~g}$ at $4^{\circ}$ C. RNA from the upper aqueous phase was extracted using the EZ1 RNA Universal Tissue Kit (Qiagen) and the EZ1 BioRobot (Qiagen) according to the manufacturer's protocol. The purified RNA was quantified using the Qubit2.0 Fluorometer with the Qubit RNA HS Assay Kit (Thermo Fisher Scientific) and the total RNA integrity was examined using the Agilent 2100 Bioanalyzer system (Agilent Technologies). Total RNA $(2 \mu \mathrm{g})$ was sent to the NGS Platform at the University of Bern (Bern, Switzerland) for Illumina HiSeq 3000150 bp paired-end sequencing.

\section{De novo genome assembly \\ De novo assembly using FALCON}

A de novo assembly of the $N$. lovaniensis genome was performed using FALCON (v.0.7.0) [14]. FALCON is a string graph assembler that uses in a first step DALIGNER [16] to compare overlapping reads and to generate high accurate consensus sequences of the longest reads with a predefined length cut off. In a second step, a string graph is constructed using the overlaps of the consensus sequences. The string graph is then reduced to primary and associative contigs representing heterozygous sequences [14]. To assemble the $N$. lovaniensis genome, the length cut off used for initial mapping was auto-calculated by setting the genome size to $30 \mathrm{Mb}$, while the length cut off for the pre-assembly was set to 5000. For the overlap filtering max_diff was set to 100 , min_cov was set to 4 , and max_cov was set to 200 to retrieve repetitive regions, the rDNA plasmid sequence and the mitochondrial sequence. To improve the genomic consensus, the assembly was polished using Quiver [17]. Additionally, the mitochondrial sequence was circularized manually.

\section{Quality assessment of the genome assembly}

To assess the completeness of the de novo assembled genome, BUSCO v2 [18] was used to search for a set of 303 conserved eukaryotic Benchmarking Universal Single-Copy Orthologs [19]. To validate the output of BUSCO and for comparison between Naegleria species, the tool was applied to the previous published genomes of $N$. fowleri and N. gruberi.

\section{Assembly and annotation of the rDNA plasmid}

Due du its high repeat content, the rDNA plasmid cloud not be fully assembled in the initial FALCON assembly. To improve the assembly, raw reads were mapped to the $12 \mathrm{~kb}$ rDNA fragment assembled by FALCON using minimap2 [20]. Mapped reads were assembled using Canu 1.7 [13] followed by Quiver polishing and manual circularization. Non-coding RNAs and rRNA sequences were annotated using INFERNAL 1.0.2 [21] and BLAST to the $N$. gruberi annotated rDNA plasmid (Accession: MG699123.1) as well as to a public available $N$. lovaniensis ribosomal sequence (Accession:MH304644).

\section{Genome annotation \\ Repetitive elements}

Repetitive elements were predicted using a de novo approach by applying RepeatModeler [22], which includes RECON [23] and RepeatScout [24] to construct a Naegleria-specific repeat library. To further classify repetitive sequences, Hmmer3.1b1 [25] was used to search for protein domains and known LTR domains. Sequences with similarities to known proteins were discarded from the repeat library. Furthermore, repetitive sequences were submitted to TEclass [26] to categorize the sequences into DNA transposons, LTRs, and non-LTRs. In a second step, RepeatMasker [27] was applied to mask repetitive sequences using the de novo constructed library.

\section{Gene annotation}

Protein coding genes on the nuclear genome were predicted using an ab-initio approach taking into account RNAseq data by applying BRAKER1 [28]. BRAKER1 uses GeneMark-ET [29] to generate ab-initio gene models by unsupervised learning including RNAseq 
data. In a second step the gene models are used to train AUGUSTUS [30]. AUGUSTUS uses unassembled RNAseq data for the final gene prediction. Non-coding RNAs were annotated by searching the Rfam 12.1 database [31] for sequence similarities using INFERNAL 1.0.2. Functional annotation of predicted proteins was done by BLAST similarity search of the predicted proteins against SwissProt and UniRef90 [32] with an e-value of 1e-05. The top five BLAST hits were loaded into CLC Genomics Workbench 9.5.2 (Qiagen) and GO terms were retrieved using Blast2GO [33] plug-in with default annotation parameters. To further improve the annotations, InterproScan was used within CLC to identify known protein domains. BLAST results of both databases and results of the InterproScan analysis were combined to gain a complete functional annotation of all predicted genes. Additionally, PFAM domain search was performed separately against PFAM-A 29.0 [34] using HMMER v3.1b2. Figure 1 depicts a summary of the de novo genome assembly and annotation workflow.

\section{Genome similarity}

To gain an overview of the taxonomic relationship of Naegleria, a BLAST-based genome similarity network was constructed using Evolutionary Gene and Genome Networks software (EGN) [35]. The similarity network contains predicted proteins of $N$. lovaniensis and TransDecoder predicted Open Reading Frames (ORFs) of $N$. fowleri [8]. Additionally, the proteomes of N. gruberi
(UP000006671), two human pathogenic amoebas, E. histolytica (UP000001926) and A. castelanii (UP000011 083), as well as T. brucei (UP000008524) and T. cruzi (UP000017861), both belonging to the related group of Euglenozoans, were retrieved from UniProt. To identify shared protein families, an all-versus-all BLAST search was performed within EGN. Similar sequences were clustered by applying an e-value of $1 \mathrm{e}-5$ and a hit identity of $50 \%$. The network was visualized using Cytoscape 3.0.2 [36].

\section{Clustering of orthologous genes}

To identify orthologs between different Naegleria species and to estimate the degree of relatedness, proteins of $N$. lovaniensis, $N$. gruberi (UP000006671) and N. fowleri were clustered using OrthoMCL [37]. For BLAST similarity search an e-value of 1e-5 and a minimal identity of $50 \%$ were applied.

\section{Phylogenetic analysis}

To construct a phylogenetic tree, random 100 single-copy orthologs of the OrthoMCL clustering were aligned using MUSCLE [38]. Alignments were trimmed using trimAl [39] and concatenated to a supermatrix using FASconCAT [40]. Prottest3 [41] was used to select the best model for amino acid replacements, the phylogenetic tree was constructed based on maximum likelihood and bootstrapping using RAxML 8.1.2 [42] including 1000 bootstraps. The resulting tree was visualized with FigTree [43].

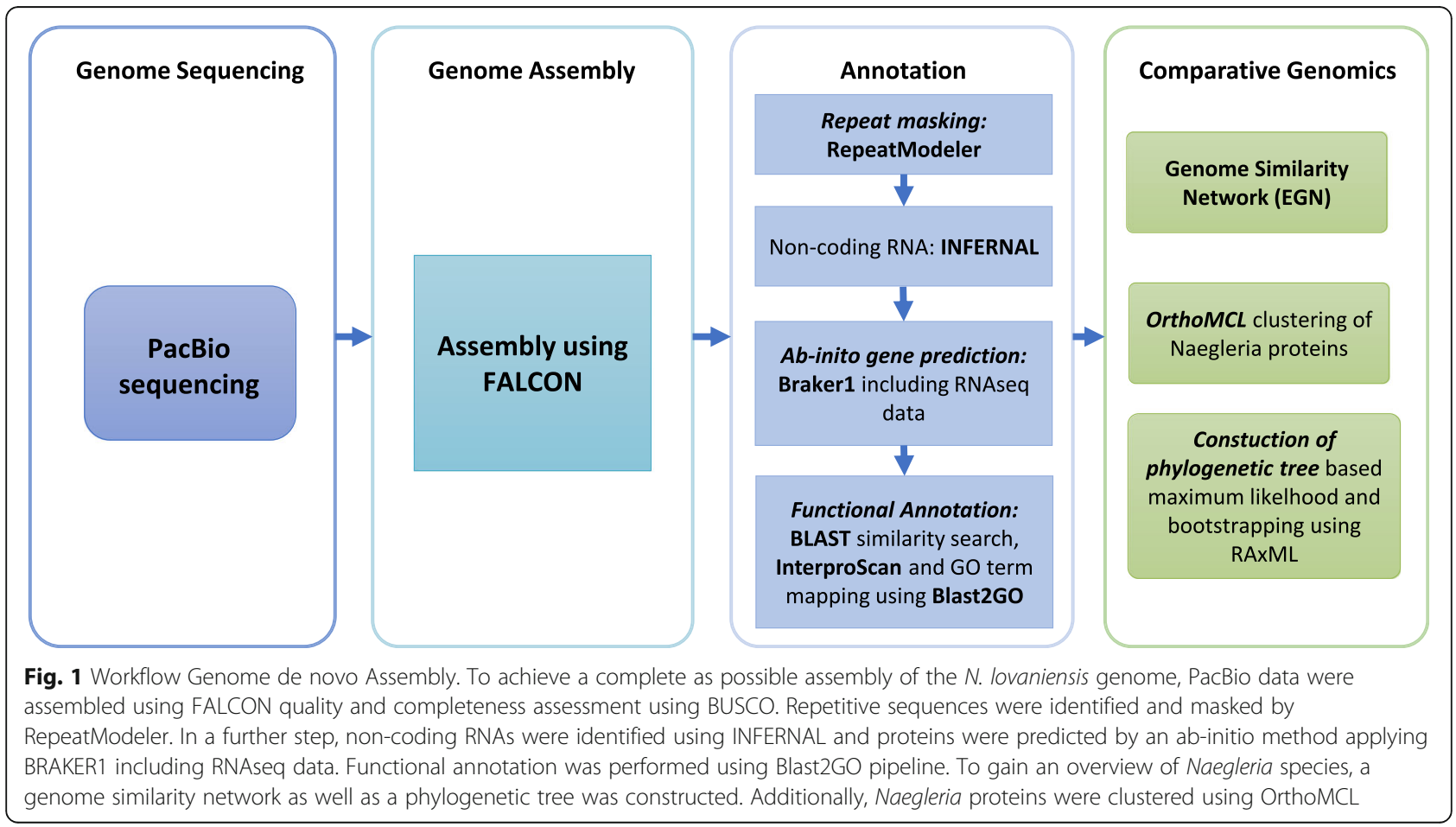




\section{Gene set enrichment analysis}

To identify the function of $N$. fowleri and $N$. lovaniensis specific proteins, GO enrichment analysis was performed using the Cytoscape plug-in BiNGO [44]. As reference set for GO enrichment analysis, all functionally annotated proteins of the respective species were considered. Over-represented GO terms (Bonferroni-corrected $p$-value $<0.05)$ were examined using classical hypergeometric test.

\section{Results}

\section{Genome assembly}

To achieve a nearly complete genome assembly, total DNA of N. lovaniensis (ATCC \#30569) was isolated and sequenced using the PacBio RSII sequencing platform. Sequencing of 10 SMRT cells resulted in 1,529,980 reads with a mean read length of $6893 \mathrm{bp}$ after subread filtering. To reconstruct the genome, the data were assembled using FALCON, a diploid-aware string-graph assembler. To improve the consensus sequence and to correct insertions, deletions and substitution errors, raw reads were realigned to the assembly and the sequence was polished using Quiver. The final haploid assembly consists of 111 contigs with an N50 of 657,933 bp and has a total size of $30.8 \mathrm{Mb}(30,838,059 \mathrm{bp})$. The genome of $N$. lovaniensis has a similar size as well as a similar GC content (37\%) compared to the recently published $N$. fowleri genome. In contrast, the $N$. gruberi genome is slightly larger $(40 \mathrm{Mb})$ and has a GC content of 35\% [9] (Table 1). The completeness of the assembly was evaluated by searching for 303 eukaryotic Benchmarking Universal Single-Copy Orthologs (BUSCOs) [18]. BUSCOs evolved as single copy orthologs in a broad range of organisms; the eukaryotic set was selected based on OrthoDB v9.1 [45] data of 90 different species. Since the duplication or deletion of such evolutionary conserved single-copy genes are rare events, analysis of BUSCOs gives an overview of the completeness of de novo assembled genomes [18]. Analysing the N. lovaniensis genome, 265 (87.4\%) BUSCOs were found in the final FALCON assembly, of which 6 are fragmented and 18 are

Table 1 Comparison of sequenced Naegleria genomes

\begin{tabular}{|c|c|c|c|}
\hline & $\begin{array}{l}N . \\
\text { lovaniensis }\end{array}$ & N. fowleri & $\begin{array}{l}N . \\
\text { gruberi }\end{array}$ \\
\hline Genome Size (Mb) & 30.8 & 29.6 & 40.9 \\
\hline GC content (\%) & 37 & 37 & 35 \\
\hline Repeat content (\%) & 3.5 & 2.5 & 5.1 \\
\hline $\begin{array}{l}\text { Number of contigs } \\
\text { (Scaffolds) }\end{array}$ & 111 & $1729(574)$ & $\begin{array}{l}1977 \\
(784)\end{array}$ \\
\hline N50 of contigs (bp) & 657,933 & 38,128 & 159,679 \\
\hline $\begin{array}{l}\text { Number of predicted } \\
\text { genes }\end{array}$ & 15,195 & $\begin{array}{l}\text { 17,252 (based on } \\
\text { RNAseq data) }\end{array}$ & 15,711 \\
\hline
\end{tabular}

duplicated. To benchmark the completeness of the assembly, the number of found BUSCOs was compared to those found in $N$. fowleri and $N$. gruberi. The comparison shows similar numbers of BUSCOs in the $N$. fowleri genome (total: 269, 88.8\%) while less BUSCOs could be identified in the N. gruberi assembly (total: $257,84.8 \%$ ) (Table 2).

\section{Genome structure}

The N. lovaniensis genome is gene dense, $77 \%$ of the genome is defined as coding sequences. Further, $47 \%$ of the predicted genes have at least one intron with a median length of $80 \mathrm{bp}$. Besides its nuclear DNA, the genome of Naegleria includes a circular mitochondrial sequence and an extrachromosomal plasmid encoding ribosomal RNAs [46]. The long-read assembly allowed the complete reconstruction of the mitochondrial genome sequence. With a size of $48,553 \mathrm{bp}$ the mitochondrial genome of $N$. lovaniensis is shorter compared to the ones of $N$. fowleri $(49,519 \mathrm{bp})$ and $N$. gruberi (49,842 bp). Furthermore, the extrachromosomal rDNA plasmid was assembled using Canu 1.7. With 15,131 kb the assembly length corresponds to the estimated size of the plasmid [47].

\section{Genome annotation}

Gene prediction in eukaryotes is still a challenge, especially in species with little information. Naegleria spp. are distantly related to other annotated species and lacking a well-established reference, de novo repeat prediction was performed using RepeatModeler to build a Naegleria-specific library. Approximately $3.4 \%$ of the total genome is classified as repetitive sequences. In total $1.25 \%$ is categorized as DNA transposons while $0.68 \%$ is classified as LTRs (Table 3). To accurately predict genes of the $N$. lovaniensis nuclear genome, an ab-initio method incorporating RNAseq data was applied. Using BRAKER1, a combination of GeneMark-ES and AUGUSTUS, 15,195 genes could be identified on the nuclear genome. In total, 13,005 (85.6\%) of the predicted proteins contain at least one Pfam protein domain. The total number of predicted proteins is slightly lower compared to $N$. gruberi $(15,727)$, while a higher number of proteins is predicted based on RNAseq data for N. fowleri $(17,252)$ [8]. By applying INFERNAL 1.0.2 for

Table 2 Comparison of BUSCO analysis of Naegleria genomes

\begin{tabular}{llll}
\hline & N. lovaniensis & N. fowleri & N. gruberi \\
\hline Total Number of BUSCOs & 265 & 269 & 257 \\
Complete Single-Copy & 247 & 265 & 253 \\
Complete Duplicated & 18 & 4 & 4 \\
Fragmented & 6 & 6 & 7 \\
Missing & 32 & 32 & 39 \\
\hline
\end{tabular}


Table 3 Repetitive Sequences of the total genome

\begin{tabular}{lllll}
\hline Class & & Count & bp Masked & $\%$ masked \\
\hline DNA & 379 & 209,650 & $0.68 \%$ \\
& CMC-EnSpm & 38 & 69,762 & $0.23 \%$ \\
& PiggyBac & 29 & 23,595 & $0.08 \%$ \\
LINE & & 190 & 78,458 & $0.25 \%$ \\
LTR & & 208 & 115,388 & $0.37 \%$ \\
& DIRS & 89 & 99,113 & $0.32 \%$ \\
& Gypsy & 22 & 52,359 & $0.17 \%$ \\
Unspecified & & 57 & 20,784 & $0.07 \%$ \\
& total interspersed & 1012 & 669,109 & $2.17 \%$ \\
Low_complexity & & 766 & 37,304 & $0.12 \%$ \\
Simple_repeat & & 7568 & 327,882 & $1.06 \%$ \\
Total & & 9346 & $1,034,295$ & $3.35 \%$ \\
\hline
\end{tabular}

non-coding RNA prediction, among others spliceosomal RNAs, U1-U6 as well as tRNA coding regions were identified. The functional annotation of the predicted proteins was performed by BLAST similarity search against SwissProt and UniRef90 by applying an e-value of 1e-05. GO terms were retrieved from BLAST results using Blast2GO CLC plug-in. The annotations were further improved using InterproScan to identify protein families. In total, 8749 (57.6\%) proteins mapped to at least one GO term, 4678 (30.8\%) only have a BLAST hit, while 1768 (11.6\%) show no similarity to known proteins or domains (Fig. 2).

\section{Genome similarity}

Unravelling the phylogenetic relationship of Naegleria is mandatory for further comparative studies on a genomic

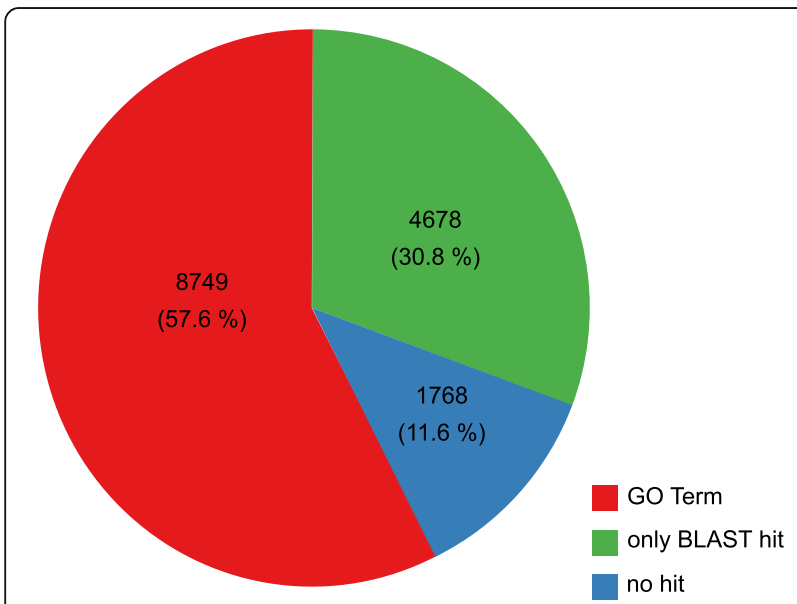

Fig. 2 Overview of gene annotation of the N. lovaniensis genome. Of total 15,195 ab initio predicted proteins, $8749(57.6 \%)$ proteins mapped to at least one GO term using Blast2GO; 4678 (30.8\%) only have a BLAST hit, while 1768 (11.6\%) show no similarity to known proteins or domains and molecular level, especially regarding the pathogenicity of $N$. fowleri. Therefore, we constructed a BLAST similarity-based genome network using Evolutionary Gene and Genome Networks software (EGN). Additional to the Naegleria species, two Trypanosoma species (T. cruzi and T. brucei), which belong to the related class Euglenozoa, and two human pathogenic amoebas (A. castellanii and E. histolytica) were included in the analysis. Within the network, nodes represent the organism and the length of the edges are proportionally inverse to the number of shared gene families. Regarding the cluster of Naegleria, N. fowleri shares 9547 genes with $N$. lovaniensis, while only 5831 genes are shared with $N$. gruberi. Furthermore, the network suggests a higher similarity between the cluster of Naegleria species and $A$. castellanii (527, N. fowleri: A. castelanii), a human pathogenic amoeba causing Granulomatous Amoebic Encephalitis, than to the trypanosomes of the related class Euglenozoa (233, N. fowleri: T. brucei) (Fig. 3, Additional file 1). To gain a comprehensive overview of the relatedness of Naegleria species, a phylogenetic tree based on maximum likelihood and bootstrapping using RAxML was constructed. Phylogenetic distances were estimated based on 100 single copy orthologs defined by OrthoMCL clustering of Naegleria species and the more distantly related protists $T$. brucei and T. cruzi as outgroups. Visualization of the tree provides a more detailed overview of the phylogenetic relationship. In concordance to the genome similarity network, the result shows a close relationship between $N$. lovaniensis and the pathogenic N. fowleri, while N. gruberi is more distantly related (Fig. 4).

\section{Clustering of gene families of Naegleria species}

To gain an overview of the protein diversity within the genus Naegleria, predicted proteins of all sequenced species were clustered into gene families based on BLAST similarities using OrthoMCL. 8114 gene families are shared among all Naegleria species and define the core genome. 2406 gene families are shared between $N$. fowleri and $N$. lovaniensis, while $N$. fowleri only shares 267 with $N$. gruberi, and $N$. lovaniensis shares 410 gene families with $N$. gruberi. Further, 191 gene families are specific for $N$. lovaniensis, 323 are specific for $N$. fowleri and 626 are specific for N. gruberi (Fig. 5).

To assess the function of protein clusters specific for $N$. fowleri in terms of pathogenicity, GO term enrichment analysis was performed using BiNGO. Regarding the category Biological Process, GO terms describing secretory processes and protein transport are enriched among $N$. fowleri specific proteins (Table 4). Further, the GO term membrane (GO:0016020, $p$ value $=1.0188 \mathrm{E}-4$ ) is significantly overrepresented in the category Cellular 


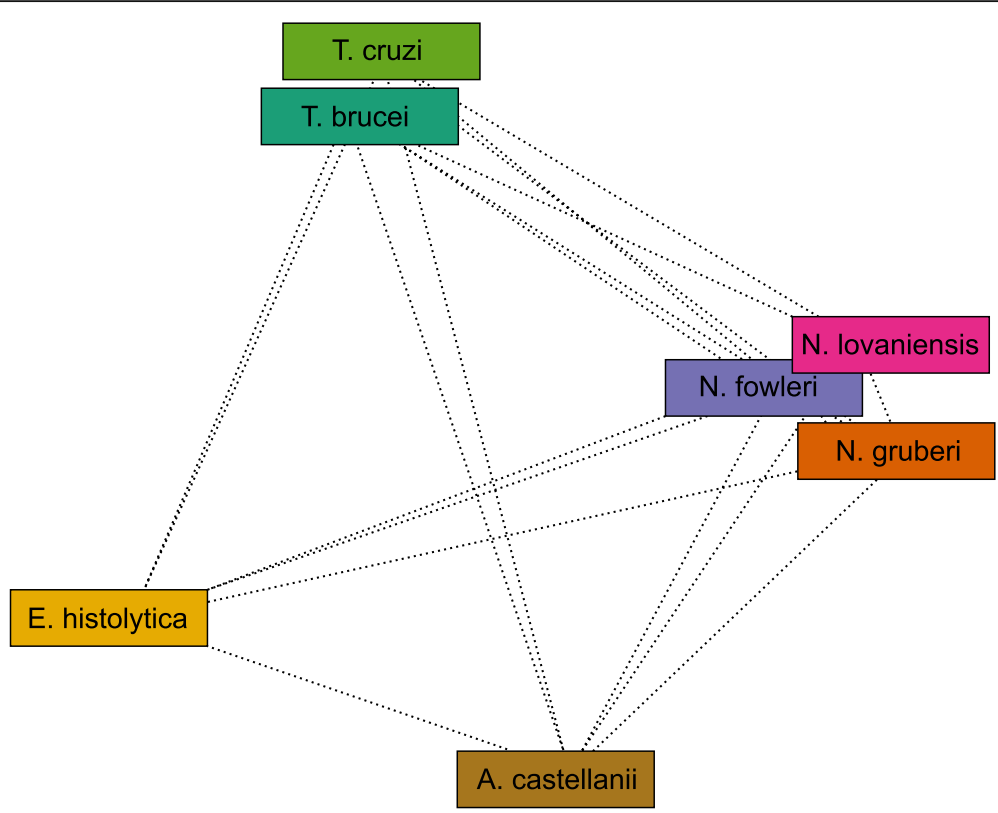

Fig. 3 Genome Similarity Network of unicellular eukaryotic species. To gain insights of the phylogenetic relationship of Naegleria, a BLAST based Genome Network was constructed including N. lovaniensis and N. fowleri predicted protein as well as Uniprot proteomes of T. brucei, T. cruzi, A. castelanii, and E. histolytica. Nodes in the graph represent the organisms and the edges, which are inversely proportional to the number of shared gene families, are the measurement of similarity between nodes. Additional file 2 shows the number of shared gene families between species

Component (Table 4). In contrast, analysing N. lovaniensis specific proteins, no significantly overrepresented term was found. In total, 97 proteins in the N. fowleri specific cluster are annotated with the GO term membrane. BLAST similarity search and PFAM Domain analysis of these proteins identified similarities to vesicular and cytoskeletal regulatory proteins including Rab and Rho small family GTPase related proteins as well as proteins containing an adenylate and guanylate cyclase catalytic domain (see Additional file 1).

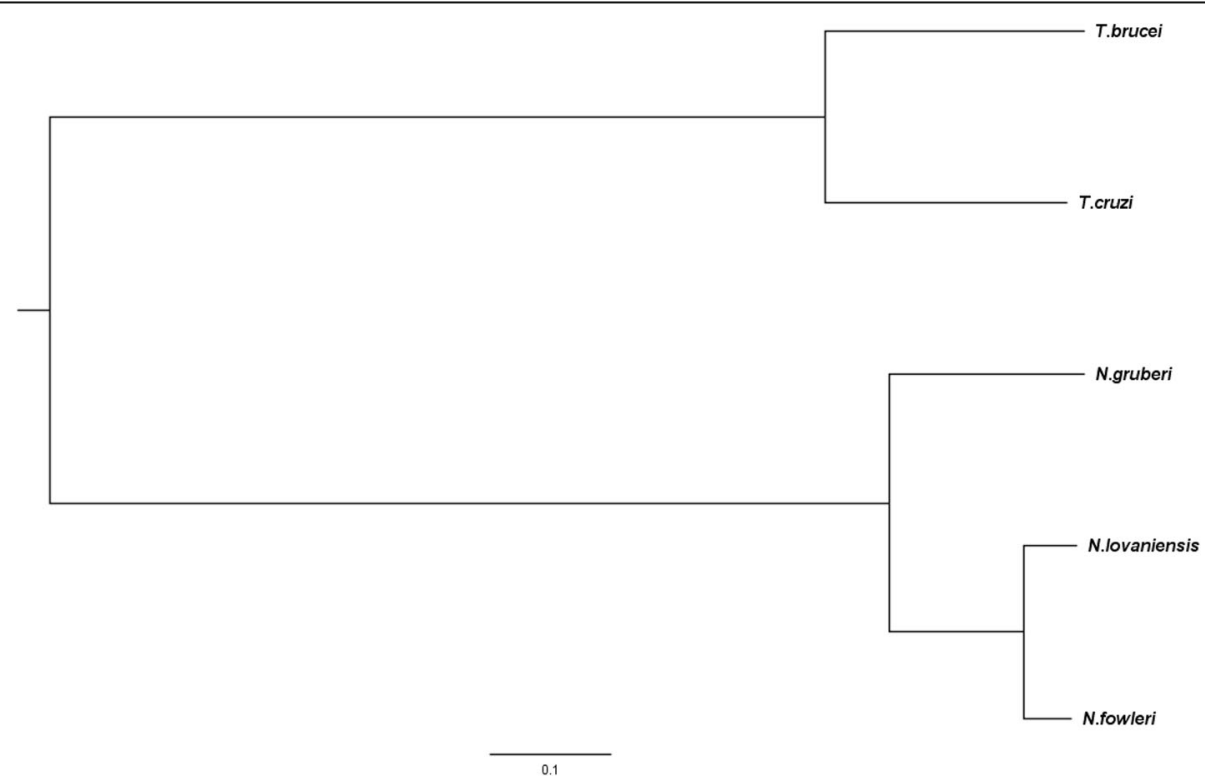

Fig. 4 Maximum likelihood tree of Naegleria species. Based on maximum likelihood and bootstrapping using RAxML a species tree was constructed to achieve a comprehensive overview of the relatedness of the species within the genus of Naegleria. Phylogenetic distances were estimated based on 100 single copy orthologs defined by OrthoMCL clustering. Beside Naegleria species, the more distantly related protists $T$. brucei and $T$. cruzi were chosen as taxonomic outgroups 


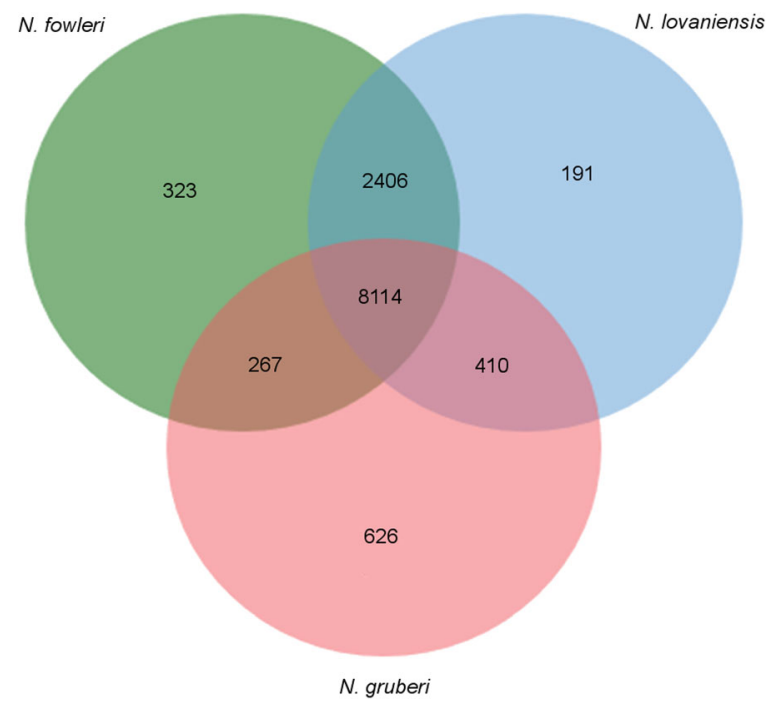

Fig. 5 OrthoMCL Clustering of Naegleria proteins. To gain an overview of the diversity of the Naegleria protein repertoire, predicted protein of $\mathrm{N}$. lovaniensis and $\mathrm{N}$. fowleri as well as protein sequences of $\mathrm{N}$. gruberi retrieved form Uniprot were clustered using OrthoMCL

\section{Discussion}

Members of the genus Naegleria belong to a species-rich group of free-living eukaryotes. Besides the diversity within the genus, this protist can adapt to unfavorable environmental conditions by transforming to resting cysts or moving flagellates. As free-living eukaryotes with different life stages including facultative pathogenic species, Naegleria shows a high plasticity regarding morphology and pathogenicity. Regarding this feature, Naegleria is a powerful model to study fundamental eukaryotic processes including pathogenesis. Over decades, more than 40 Naegleria species were characterized based on ribosomal sequences, but large scale genomic approaches to unravel phylogenetic relationships are still missing [3, 48]. Here, we present the genome of $N$. lovaniensis, the closest relative of the human pathogenic amoeba $N$. fowleri. Assembling of PacBio data resulted in a nearly complete assembly of the $N$. lovaniensis genome. Based on the analysis of BUSCOs, the completeness of the assembly is comparable to the $N$. fowleri genome published in 2014. Furthermore, the assembly of $N$. lovaniensis is, with only 112 contigs, much less fragmented. Using the string graph assembler FALCON, we were able to fully assemble and circularize the mitochondrial sequence. Analysis of the mitochondrial gene repertoire shows high similarity with other annotated mitochondrial sequences within the genus of Naegleria. A typical Naegleria genome contains about 3000-5000 copies of an extrachromosomal rDNA plasmid [49]. Using long reads, the $15 \mathrm{~kb}$ rDNA plasmid could be reconstructed and rRNA genes were annotated. To summarize, the de novo sequenced genome of $N$. lovaniensis contains all characteristics of previous sequenced Naegleria species. By applying ab-initio gene prediction methods including RNAseq data, 15,195 proteins could be annotated and to 8749 (57.6\%) at least one GO Term was mapped using Blast2GO annotation pipeline. A comparable number of predicted proteins is found in the $N$. gruberi genome. Proteins of $N$. fowleri were predicted based on transcriptome de-novo assembly using Trinity and TransDecoder to extract ORFs [8]. Trinity reports for each gene different isoforms, therefore predicted proteins based on TransDecoder may contain clusters of trinity isoforms. Further, TransDecoder is not considering start and stop codons. Therefore, the set of predicted proteins may contain incomplete proteins explaining the higher number reported for $N$. fowleri. With the description of the $30 \mathrm{Mb}$ genome of $N$. lovaniensis, a further piece is added to reveal the complex taxonomic relationship of Naegleria. Using BLAST similarity genome networks and construction of a phylogenetic tree based on OrthoMCL single-copy orthologs, we confirm the close relationship of $N$. lovaniensis and $N$. fowleri. Within the genome similarity network, the Naegleria species share more genes with the human pathogenic amoeba $A$. castelanii than the closely related trypanosomes. Compared to free-living eukaryotes, obligate parasites including trypanosomes, show a functional reduction of their gene repertoire in adaption to the life cycle in the host,

Table 4 GO Enrichment of N. fowleri Specific Proteins (adj. p-value <0.05) BiNGO

\begin{tabular}{lll}
\hline GO ID & Name & Adj. P-value \\
\hline Biological Process & & $4.1060 \mathrm{E}-2$ \\
GO:0009235 & cobalamin metabolic process \\
G0:0050708 & regulation of protein secretion & $4.1060 \mathrm{E}-2$ \\
G0:0050709 & negative regulation of protein secretion & $4.1060 \mathrm{E}-2$ \\
G0:0051224 & negative regulation of protein transport & $4.1060 \mathrm{E}-2$
\end{tabular}

Cellular Compartment

GO:0016020 
resulting for example in reduced metabolic capabilities [50]. The higher total number of $A$. castelanii proteins as well as functional protein similarities within free-living amoebas may explain the higher number of shared genes between $A$. castelanii and Naegleria in the genome similarity network. Based on the genomic data presented in this study and previous ribosomal sequence analysis, it is plausible that $N$. fowleri and $N$. lovaniensis evolved from a recent common ancestor $[2,51]$. Due to the high similarity and the close relationship of the amoebas, knowledge of the $N$. lovaniensis genome provides the basis for further comparative studies to unravel pathogenicity factors of the human pathogenic $N$. fowleri. Transcriptomics. To gain insights in cellular pathways specific to $N$. fowleri and their involvement in pathogenesis, a closer look at $N$. fowleri specific proteins within the OrthoMCL clustering was taken. Characterizing the function of these proteins using GO enrichment analysis highlights the importance of the term membrane (GO:0016020) and the secretory protein system (GO:0050708, GO:0050709, GO:0051224). In concordance to our results, a comparative study of $N$. fowleri trophozoites with difference in pathogenicity levels shows an association of overexpressed proteins in highly pathogenic amoebas with the GO terms membrane and vesicles [8]. Already around 1990 N. fowleri has been compared to N. lovaniensis and other non-pathogenic Naegleria species to characterize pathogenicity factors. In contrast to weakly pathogenic $N$. australiensis and non-pathogenic $N$. lovaniensis, $N$. fowleri is more resistant to complement lysis when incubated with human serum [52]. Furthermore, only N. fowleri, but not N. gruberi shows membrane vesiculation during contact with human serum to eliminate MAC complex on its membrane [53]. A further comparison of $N$. fowleri with non-pathogenic Naegleria species and Acanthamoeba species identified a membrane protein (Mp2CL5), which is unique for $N$. fowleri and not expressed in other Naegleria species. It is localized at pseudopod structures and likely involved in attachment or sensing of nutrient and other external environmental influences [54]. Comparing the adhesion to extracellular matrix structures of pathogenic and non-pathogenic Naegleria shows a strong attachment of $N$. fowleri while a weaker interaction is reported for $N$. lovaniensis. In addition, the formation of focal adhesion structures containing actin and integrin-like proteins is observed in $N$. fowleri but not for N. lovaniensis. Furthermore, a $70 \mathrm{kDa}$ protein species was found to be expressed at higher levels ins $N$. fowleri [55]. Attachment to the host cell is a crucial point in the pathogenesis of $N$. fowleri. In this context, an integrin-like membrane protein binding to fibronectin was identified and the formation of actin structures regulated by protein kinase $\mathrm{C}$ in response to fibronectin was shown in $N$. fowleri trophozoites [56]. Altogether, expression of membrane proteins and regulation of membrane structures either involved in attachment to the host membrane or defending against lysis, are important mechanisms of pathogenesis. Vesicular transport and regulation of protein secretion are important points in the pathogenesis of $N$. fowleri and is supported by the GO enrichment analysis. Beside the above mentioned membrane vesiculation, recent studies identified secreted cysteine and metalloproteinases which are involved in the degradation of the host extracellular matrix and enable the penetration of the blood-brain barrier [57-59].

Among others, BLAST similarity search of $N$. fowleri specific proteins associated with the GO term membrane identified different Rab and Rho family small GTPase proteins. Furthermore, a recent proteomics study identified an upregulation of the Rho guanine nucleotide exchange factor 28 , which is involved in actin regulation, in highly pathogenic $N$. fowleri [60]. Cytoskeleton changes and the formation of actin-rich structures in response to extracellular matrix protein has also been reported for E. histolytica. There, adhesion to fibronectin may induce formation of further attachment structures and the secretion of proteolytic enzymes [61]. A recent study in E. histolytica shows the role of Rab21-mediated actin cytoskeleton changes and the involvement of the Rab GTPase in the formation of actin dots during invasion of the extracellular matrix [62]. Rab GTPases are not only known as the master regulators in vesicular transport but are also involved in the regulation of actin cytoskeleton changes. Regarding the pathogenicity of $N$. fowleri, Rab GTPases might not only play an important role in the regulation of vesicular transport and secretion of proteases but also in the formation of actin structures promoting adhesion to the host cell.

\section{Conclusion}

Sequencing and de novo assembly of the N. lovaniensis genome supports the hypothesis of the close relationship to the human pathogenic amoeba $N$. fowleri. Thus, knowledge of the $N$. lovaniensis genome provides the basis for a more detailed interspecies comparison on a genomic, proteomic or molecular level to unravel pathways involved in the pathogenicity of PAM and to identify potential structures for possible treatment options.

\section{Additional files}

Additional file 1: $N$. fowleri specific Proteins associated with the GO Term "Membrane". Results of PFAM domain analysis and BLAST search against SwissProt of $N$. fowleri specific proteins associated with the GO Term "Membrane". (XLSX 17 kb)

Additional file 2: Genome Similarity Network: Number of shared gene families between unicellular eukaryotic species within the Genome Similarity Network. (XLSX 9 kb) 


\section{Abbreviations}

BUSCO: Benchmarking Universal Single-Copy Ortholog; EGN: Evolutionary Gene and Genome Network; GO: Gene Ontology; LTRs: Long-terminal Repeat; NGS: Next Generation Sequencing; ORF: Open Reading frame: PAM: Primary amoebic meningoencephalitis; PBS: Phosphate-buffered saline; RNAseq: RNA sequencing

\section{Acknowledgements}

We thank the FGCZ (University of Zurich) for whole genome sequencing and the Next Generation Sequencing Platform (University of Bern) for RNA sequencing. Further, our thanks go to Heidrun Gundlach for helpful discussion concerning repeat annotation, Denise C. Zysset-Burri for protocols of Naegleria cultivation, Susanne Thomann and Sandra Paniga Rudolf for support during laboratory work, the members of the Biology Division of the Spiez Laboratory as well as the members of the Interfaculty Bioinformatic Unit (University of Bern) for their support and valuable discussions.

\section{Funding}

The study was funded by the Federal Office for Civil Protection (project number 353005702)

\section{Availability of data and materials}

This Whole Genome Shotgun project has been deposited at DDBJ/ENA/ GenBank under the accession PYSW00000000. The version described in this paper is version PYSW01000000. The PacBio raw data have been deposited in DDBJ/EMBL/GenBank under accession SRP136861.

\section{Authors' contributions}

MW and NS designed and initiated the study. NL carried out the experiment, analyzed the data and wrote the manuscript with support of RB, MW and NS. All authors read and approved the final manuscript.

\section{Ethics approval and consent to participate}

Not applicable

\section{Consent for publication}

Not applicable

\section{Competing interests}

The authors declare that they have no competing interests.

\section{Publisher's Note}

Springer Nature remains neutral with regard to jurisdictional claims in published maps and institutional affiliations.

\section{Author details}

'Interfaculty Bioinformatics Unit, University of Bern, Bern, Switzerland. ${ }^{2}$ Biology Division, Spiez Laboratory, Federal Office for Civil Protection, Austrasse, Spiez, Switzerland. ${ }^{3}$ Graduate School for Cellular and Biomedical Sciences, University of Bern, Bern, Switzerland.

\section{Received: 18 April 2018 Accepted: 3 August 2018}

\section{Published online: 05 September 2018}

\section{References}

1. Schuster FL, Visvesvara GS. Free-living amoebae as opportunistic and non-opportunistic pathogens of humans and animals. Int J Parasitol. 2004;34:1001-27.

2. De Jonckheere JF. What do we know by now about the genus Naegleria? Exp Parasitol. 2014;145:S2-9.

3. De Jonckheere JF. Molecular definition and the ubiquity of species in the genus Naegleria. Protist. 2004;155:89-103.

4. Marciano-Cabral F, Cabral $\mathrm{G}$ a. The immune response to Naegleria fowleri amebae and pathogenesis of infection. FEMS Immunol Med Microbiol. 2007:51:243-59.

5. Khan NA. Is ritual cleansing a missing link between fatal infection. Clin Infectous Dis. 2012;54:1817-8

6. Visvesvara GS, Moura H, Schuster FL. Pathogenic and opportunistic free-living amoebae: Acanthamoeba spp., Balamuthia mandrillaris, Naegleria fowleri, and Sappinia diploidea. FEMS Immunol Med Microbiol. 2007;50:1-26.
7. Sriram R, Noman F, Ali F. Primary amebic meningoencephalitis caused by Naegleria fowleri, Karachi, Pakistan. Emerg Infect Dis. 2011; 17:258-61.

8. Zysset-Burri DC, Müller N, Beuret C, Heller M, Schürch N, Gottstein B, et al. Genome-wide identification of pathogenicity factors of the free-living amoeba Naegleria fowleri. BMC Genomics. 2014;15:496.

9. Fritz-Laylin LK, Prochnik SE, Ginger ML, Dacks JB, Carpenter ML, Field MC, et al. The genome of Naegleria gruberi illuminates early eukaryotic versatility. Cell. 2010;140:631-42.

10. Cervantes-Sandoval I, Serrano-Luna JJ, Pacheco-Yépez J, Silva-Olivares A, Tsutsumi V, Shibayama M. Differences between Naegleria fowleri and Naegleria gruberi in expression of mannose and fucose glycoconjugates. Parasitol Res. 2010;106:695-701.

11. Serrano-Luna J, Cervantes-Sandoval I, Tsutsumi V, Shibayama M. A biochemical comparison of proteases from pathogenic Naegleria fowleri and non-pathogenic Naegleria gruberi. J Eukaryot Microbiol. 2007;54: 411-7.

12. Herman EK, Greninger AL, Visvesvara GS, Marciano-Cabral F, Dacks JB, Chiu CY. The Mitochondrial Genome and a 60-kb Nuclear DNA Segment from Naegleria fowleri, the Causative Agent of Primary Amoebic Meningoencephalitis. J Eukaryot Microbiol. 2013;60:179-91.

13. Koren S, Walenz BP, Berlin K, Miller JR, Bergman NH, Phillippy AM. Canu: scalable and accurate long-read assembly via adaptive $k$-mer weighting and repeat separation. Genome Res. 2017:27:722-36.

14. Chin C-S, Peluso P, Sedlazeck FJ, Nattestad M, Concepcion GT, Clum A, et al. Phased diploid genome assembly with single-molecule real-time sequencing. Nat Methods. 2016;13:1050-4.

15. Burri DC, Gottstein B, Zumkehr B, Hemphill A, Schürch N, Wittwer M, et al. Development of a high- versus low-pathogenicity model of the free-living amoeba Naegleria fowleri. Microbiology. 2012;158:2652-60.

16. Myers G. Efficient Local Alignment Discovery amongst Noisy Long Reads. In: Brown D, Morgenstern B, editors. Algorithms in Bioinformatics. WABI 2014. Springer, Berlin, Heidelberg. Lect. Notes Comput. Sci. 2014:8701:52-67.

17. Chin C-S, Alexander DH, Marks P, Klammer AA, Drake J, Heiner C, et al. Nonhybrid, finished microbial genome assemblies from long-read SMRT sequencing data. Nat Methods. 2013;10:563-9.

18. Simão FA, Waterhouse RM, loannidis $P$, Kriventseva EV , Zdobnov EM. BUSCO: assessing genome assembly and annotation completeness with single-copy orthologs. Bioinformatics. 2015;31:3210-2.

19. Simão FA, Waterhouse RM, loannidis P, Kriventseva E V., Zdobnov EM. Eukaryota Dataset ODB9. 2016. Busco.ezlab.org/datasets/eukaryote_odb9.tar. gz. Accessed 04 Nov 2016.

20. Li, H. Minimap2: pairwise alignment for nucleotide sequences Bioinformatics. 2018; https://doi.org/10.1093/bioinformatics/bty191

21. Nawrocki EP, Kolbe DL, Eddy SR. Infernal 1.0: inference of RNA alignments, Bioinformatics. 2009:25:1335-7.

22. Smit AFA, Hubley R. RepeatModeler Open-1.0. RepeatModeler Open-1.0.8. 2015. http://www.repeatmasker.org

23. Bao Z, Eddy SR. Automated de novo identification of repeat sequence families in sequenced genomes. Genome Res. 2002:12:1269-76.

24. Price AL, Jones NC, Pevzner PA. De novo identification of repeat families in large genomes. Bioinformatics. 2005:21:i351-8.

25. Eddy SR. Accelerated Profile HMM Searches. PLoS Comput Biol. 2011; 7:e1002195

26. Abrusán G, Grundmann N, DeMester L, Makalowski W. TEclass--a tool for automated classification of unknown eukaryotic transposable elements. Bioinformatics. 2009;25:1329-30.

27. Smit A, Hubley R, Green P. RepeatMasker Open-4.0. 2013-2015. http://www. repeatmasker.org.

28. Hoff KJ, Lange S, Lomsadze A, Borodovsky M, Stanke M. BRAKER1: unsupervised RNA-Seq-based genome annotation with GeneMark-ET and AUGUSTUS: table 1. Bioinformatics. 2016;32:767-9.

29. Lomsadze A, Burns PD, Borodovsky M. Integration of mapped RNA-Seq reads into automatic training of eukaryotic gene finding algorithm. Nucleic Acids Res. 2014:42:e119.

30. Stanke M, Diekhans M, Baertsch R, Haussler D. Using native and syntenically mapped CDNA alignments to improve de novo gene finding. Bioinformatics. 2008;24:637-44.

31. Griffiths-Jones S. Rfam: an RNA family database. Nucleic Acids Res. 2003; 31:439-41. 
32. Bateman A, Martin MJ, O'Donovan C, Magrane M, Alpi E, Antunes R, et al. UniProt: the universal protein knowledgebase. Nucleic Acids Res. 2017:45:D158-69.

33. Conesa A, Götz S, García-Gómez JM, Terol J, Talón M, Robles M. Blast2GO: a universal tool for annotation, visualization and analysis in functional genomics research. Bioinformatics. 2005;21:3674-6.

34. Finn RD, Coggill P, Eberhardt RY, Eddy SR, Mistry J, Mitchell AL, et al. The Pfam protein families database: towards a more sustainable future. Nucleic Acids Res. 2016;44:D279-85.

35. Halary S, Mclnerney JO, Lopez P, Bapteste E. EGN: a wizard for construction of gene and genome similarity networks. BMC Evol Biol. 2013;13:146.

36. Shannon P. Cytoscape: a software environment for integrated models of biomolecular interaction networks. Genome Res. 2003;13:2498-504.

37. Li L, Stoeckert CJ, Roos DS. OrthoMCL: identification of Ortholog groups for eukaryotic genomes. Genome Res. 2003;13:2178-89.

38. Edgar RC. MUSCLE: multiple sequence alignment with high accuracy and high throughput. Nucleic Acids Res. 2004;32:1792-7.

39. Capella-Gutiérrez S, Silla-Martínez JM, Gabaldón T. trimAl: A tool for automated alignment trimming in large-scale phylogenetic analyses. Bioinformatics. 2009;25:1972-3.

40. Kück P, Meusemann K. FASconCAT: convenient handling of data matrices. Mol Phylogenet Evol. 2010;56:1115-8.

41. Darriba D, Taboada GL, Doallo R, Posada D. ProtTest-HPC: Fast Selection of Best-Fit Models of Protein Evolution. In: Guarracino MR. et al., editors. EuroPar 2010 Parallel Processing Workshops. Euro-Par 2010. Springer, Berlin, Heidelberg. Lect. Notes Comput. Sci. 2011;6586:177-84.

42. Stamatakis A. RAxML version 8: a tool for phylogenetic analysis and postanalysis of large phylogenies. Bioinformatics. 2014;30:1312-3.

43. Rambaut A. FigTree v1. 4.0. A graphical viewer of phylogenetic trees. 2016. http://tree.bio.ed.ac.uk/software/figtree.

44. Maere S, Heymans K, Kuiper M. BiNGO: a Cytoscape plugin to assess overrepresentation of gene ontology categories in biological networks. Bioinformatics. 2005;21:3448-9.

45. Zdobnov EM, Tegenfeldt F, Kuznetsov D, Waterhouse RM, Simão FA loannidis $P$, et al. OrthoDB v9.1: cataloging evolutionary and functional annotations for animal, fungal, plant, archaeal, bacterial and viral orthologs. Nucleic Acids Res. 2017;45:D744-9.

46. Fritz-Laylin LK, Ginger ML, Walsh C, Dawson SC, Fulton C. The Naegleria genome: a free-living microbial eukaryote lends unique insights into core eukaryotic cell biology. Res Microbiol. 2011;162:607-18.

47. Clark CG, Cross GAM, De Jonckheere JF. Evaluation of evolutionary divergence in the genus Naegleria by analysis of ribosomal DNA restriction enzyme patterns. Mol Biochem Parasitol. 1989;34:281-96.

48. De Jonckheere JF. A century of research on the Amoeboflagellate genus Naegleria. Acta Protozool. 2002;41:309-42.

49. Clark CG, Cross GA. rRNA genes of Naegleria gruberi are carried exclusively on a 14-kilobase-pair plasmid. Mol Cell Biol. 1987;7:3027-31.

50. Jackson AP. Genome evolution in trypanosomatid parasites. Parasitology. 2015;142:S40-56.

51. De Jonckheere JF. Origin and evolution of the worldwide distributed pathogenic amoeboflagellate Naegleria fowleri. Infect Genet Evol. 2011; 11:1520-8.

52. Whiteman LY, Marciano-Cabral F. Susceptibility of pathogenic and nonpathogenic Naegleria spp to complement-mediated lysis. Infect Immun. 1987;55:2442-7.

53. Toney DM, Marciano-Cabral F. Membrane vesiculation of Naegleria fowleri amoebae as a mechanism for resisting complement damage. J Immunol. 1994;152:2952-9.

54. Réveiller FL, Suh SJ, Sullivan K, Cabanes PA, Marciano-Cabral F. Isolation of a unique membrane protein from Naegleria fowleri. J Eukaryot Microbiol. 2001;48:676-82.

55. Jamerson M, da Rocha-Azevedo B, Cabral GA, Marciano-Cabral F. Pathogenic Naegleria fowleri and non-pathogenic Naegleria lovaniensis exhibit differential adhesion to, and invasion of, extracellular matrix proteins. Microbiology. 2012;158:791-803.

56. Han KL, Lee HJ, Myeong HS, Shin HJ, Im Kl, Park SJ. The involvement of an integrin-like protein and protein kinase $\mathrm{C}$ in amoebic adhesion to fibronectin and amoebic cytotoxicity. Parasitol Res. 2004;94:53-60.

57. Vyas IK, Jamerson M, Cabral G a, Marciano-Cabral F. Identification of peptidases in highly pathogenic vs. weakly pathogenic Naegleria fowleri Amebae. J Eukaryot Microbiol. 2015;62:51-9.
58. Lam C, Jamerson M, Cabral G, Carlesso AM, Marciano-Cabral F. Expression of matrix metalloproteinases in Naegleria fowleri and their role in invasion of the central nervous system. Microbiology. 2017;163:1436-44.

59. Coronado-Velázquez D, Betanzos A, Serrano-Luna J, Shibayama M. An in vitro model of the blood-brain barrier: Naegleria fowleri affects the tight junction proteins and activates the microvascular endothelial cells. J Eukaryot Microbiol. 2018;(0):1-16.

60. Jamerson M, Schmoyer JA, Park J, Marciano-Cabral F, Cabral GA. Identification of Naegleria fowleri proteins linked to primary amoebic meningoencephalitis. Microbiology. 2017;163:322-32.

61. Meza I. Extracellular matrix-induced signaling in entamoeba histolytica: its role in invasiveness. Parasitol Today. 2000;16:23-8.

62. Emmanuel M, Nakano YS, Nozaki T, Datta S. Small GTPase Rab21 mediates fibronectin induced actin reorganization in Entamoeba histolytica: implications in pathogen invasion. PLoS Pathog. 2015;11:e1004666.

\section{Ready to submit your research? Choose BMC and benefit from:}

- fast, convenient online submission

- thorough peer review by experienced researchers in your field

- rapid publication on acceptance

- support for research data, including large and complex data types

- gold Open Access which fosters wider collaboration and increased citations

- maximum visibility for your research: over $100 \mathrm{M}$ website views per year

At BMC, research is always in progress.

Learn more biomedcentral.com/submissions 